\title{
Genome-wide identification of long non-coding RNAs in tomato plants irradiated by neutrons followed by infection with Tomato yellow leaf curl virus
}

Yujie Zhou $^{1}$, Won Kyong Cho ${ }^{2}$, Hee Seong Byun ${ }^{3}$, Vivek Chavan ${ }^{1}$, Eui-Joon Kil ${ }^{3}$, Sukchan Lee ${ }^{\text {Corresp., }}{ }^{3}$, Seung-Woo Hong ${ }^{\text {Corresp. } 4}$

1 Department of Energy Science, Sungkyunkwan University, Suwon, South Korea

3 Department of Genetic Engineering, Sungkyunkwan University, Suwon, South Korea

4 Department of Physics, Sungkyunkwan University, Suwon, South Korea

Corresponding Authors: Sukchan Lee, Seung-Woo Hong

Email address: cell4u@skku.edu, swhong@skku.ac.kr

Long non-coding RNAs (IncRNAs) play an important role in regulating many biological processes. In this study, tomato seeds were first irradiated by neutrons. Eight tomato mutants were then selected and infected by Tomato yellow leaf curl virus (TYLCV). RNA sequencing followed by bioinformatics analyses identified 1,563 tomato IncRNAs. About half of the IncRNAs were derived from intergenic regions, whereas antisense IncRNAs accounted for $35 \%$. There were fewer IncRNAs identified in our study than in other studies identifying tomato IncRNAs. Functional classification of 794 IncRNAs associated with tomato genes showed that many IncRNAs were associated with binding functions required for interactions with other molecules and localized in the cytosol and membrane. In addition, we identified 19 up-regulated and 11 down-regulated tomato IncRNAs by comparing TYLCV infected plants to non-infected plants using previously published data. Based on these results, the IncRNAs identified in this study provide important resources for characterization of tomato IncRNAs in response to TYLCV infection. 
1 Genome-wide identification of long non-coding RNAs in tomato plants irradiated by

2 neutrons followed by infection with Tomato yellow leaf curl virus

3

4 Yujie Zhou 1,*, Won Kyong Cho ${ }^{2, *}$, Hee-Seong Byun ${ }^{3}$, Vivek Chavan ${ }^{4}$, Eui-Joon Kil ${ }^{3}$,

5 Sukchan Lee ${ }^{3, \#}$, and Seung-Woo Hong ${ }^{4, \#}$

6

7 1Department of Energy Science, Sungkyunkwan University, Suwon 16419, Republic of Korea

$8{ }^{2}$ Research Institute of Agriculture and Life Sciences, College of Agriculture and Life Sciences,

9 Seoul National University, Seoul 08826, Republic of Korea

$10{ }^{3}$ Department of Genetic Engineering, Sungkyunkwan University, Suwon 16419, Republic of

11 Korea

$12{ }^{4}$ Department of Physics, Sungkyunkwan University, Suwon 16419, Republic of Korea

14 "These authors contributed equally to this work.

\section{Corresponding authors:}




\section{ABSTRACT}

Long non-coding RNAs (lncRNAs) play an important role in regulating many biological processes. In this study, tomato seeds were first irradiated by neutrons. Eight tomato mutants were then selected and infected by Tomato yellow leaf curl virus (TYLCV). RNA sequencing followed by bioinformatics analyses identified 1,563 tomato lncRNAs. About half of the lncRNAs were derived from intergenic regions, whereas antisense lncRNAs accounted for $35 \%$. The number of identified lncRNAs in our study was less than those of other previous studies identifying tomato lncRNAs. Functional classification of 794 lncRNAs associated with tomato genes showed that many lncRNAs were associated with binding functions required for interactions with other molecules and localized in the cytosol and membrane. In addition, we identified 19 up-regulated and 11 down-regulated tomato lncRNAs by comparing TYLCV infected plants to non-infected plants using previous published data. Based on these results, the lncRNAs identified in this study provide important resources for characterization of tomato lncRNAs in response to TYLCV 40 infection.

\section{Keywords:}

43 Long non-coding RNA, next-generation sequencing, neutron irradiation, tomato, TYLCV 44 infection, RNA-Seq 


\section{INTRODUCTION}

Several of the numerous RNAs that are transcribed by plant genomes, such as messenger RNAs, are translated into proteins. However, others without coding capacity, including non-coding RNAs (ncRNAs), are abundantly present in plant cells (Fatica \& Bozzoni 2014). Long non-coding RNAs (lncRNAs) are defined as non-coding RNAs longer than 200 nucleotides (nt) (Mercer et al. 2009). Due to the rapid advance of next-generation sequencing (NGS) techniques and bioinformatics tools, a large number of lncRNAs have been identified from various organisms such as animals and plants (Sun et al. 2017b). However, lncRNAs have been identified in a limited number of plant species such as Arabidopsis thaliana, medicago, potato, rice, strawberry, tomato (Solanum lycopersicum), and maize (Guo \& Liu 2017; Kang et al. 2017; Nejat \& Mantri 2017; Scarano et al. 2017; Zheng et al. 2017; Zhu et al. 2017). Furthermore, studies revealing functional roles of identified lncRNAs in plants are rare. A few studies have shown that lncRNAs might be involved in regulation of several biological functions, such as scaffolding of multiple proteins and gene expression (Ransohoff et al. 2017). In addition, some studies have demonstrated the involvement of plant lncRNAs in biotic and abiotic stresses. For example, previous studies using strand-specific RNA-sequencing has identified several lncRNAs in Arabidopsis (Zhu et al. 2014) and banana (Li et al. 2017), which might be responsible to infection of Fusarium oxysporum. Another study has identified DROUGHT INDUCED lncRNA (DRIR) from Arabidopsis, which plays a role in responses of drought and salt stress (Qin et al. 2017).

Plant transcriptomes can be affected by diverse environmental stimuli such as biotic and abiotic stresses (Nejat \& Mantri 2017). Of the known and diverse irradiations, neutron irradiation can have an effect on the genome and transcriptome of living organisms. Furthermore, there are thousands of low energy neutrons in our natural environment, most originating from cosmogenic 
68 neutron irradiation (Lal 1987). A short duration of cosmogenic neutron irradiation does not

69 significantly damage living organisms; however, accumulated neutron radiation over a long time

70

71

72

73

74

75

76

77

78

79

80

81

82

could be harmful (Bowlt 1994). Recently, neutron radiation has been used to generate deletion mutant populations in diverse plant species, such as barley, rice, pepper, sesame, and Arabidopsis, due to its efficient mutagenesis (Ahloowalia \& Maluszynski 2001). In addition, those neutron radiation contributes to the evolution of plants on the earth. Changes of plant genomes by neutron irradiation is not always harmful. Sometimes, mutations caused by neutron irradiation in a plant could provide the resistance against a specific biotic stress such as virus infection.

The tomato is an economically important crop as well as a model plant for plant science. Tomato yellow leaf curl virus (TYLCV) is one of the serious pathogens causing heavy economical losses. TYLCV in the genus Begomovirus is a circular DNA virus and has a broad range of hosts (Moriones \& Navas-Castillo 2000; Polston \& Lapidot 2007). To date, many studies have conducted to find tomato cultivars resistance to identify resistance genes to TYLCV (Ji et al. 2007; Ji et al. 2009; Zamir et al. 1994). Furthermore, a few recent studies have shown that some lncRNAs are involved in TYLCV defense mechanisms in the tomato. For instance, two different lists of lncRNAs associated TYLCV infection have been identified from resistant and susceptible tomato plants, respectively (Wang et al. 2018a; Wang et al. 2015).

In this study, mutagenesis was performed on tomato seeds by neutron irradiation. The irradiated tomato plants were infected by TYLCV to select tomato mutants showing resistance against TYLCV infection. To reveal the functional roles of lncRNAs against TYLCV infection in neutron irradiated tomato plants, we conducted RNA-Sequencing (RNA-Seq) for the eight selected tomato mutants. As a result, we identified 1,563 tomato lncRNAs using RNA-Seq and bioinformatics analyses. Furthermore, we characterized the functional roles of the identified 
91 lncRNAs and revealed differentially expressed lncRNAs in response to TYLCV infection using

92 public available data.

94 MATERIALS AND METHODS

95 Neutron irradiation, plant growth, and TYLCV infection

96 In this study, we used seeds of the tomato cultivar 'Seokwang,' which is susceptible to TYLCV,

97 for neutron irradiation. Two different seed conditions, dry and presoaked, were subjected to

98 neutron irradiation using the MC_50 cyclotron at the Korea Institute of Radiological and Medical

99 Science (KIRAMS) in Seoul, Korea. The presoaked seeds contain a higher portion of oxygen and

100 hydrogen as compared to dry seeds. In particular, it is known that the oxygen can interact with the

101 neutron during irradiation process producing the rapid reactive oxygen species (ROS). ROS plays

102 a pivotal role as a signaling molecule in plants involved in pathogen defense (Apel \& Hirt 2004).

103 Thus, it will be interesting to examine possible effects of ROS in the plant transcriptome. The

104 tomato seeds were subjected to neutron irradiation by proton bombardment of beryllium at 40

$105 \mathrm{MeV}$ energy and a $20 \mu \mathrm{A}$ current. Two different irradiation times of $30 \mathrm{~min}$ and 90 min were

106 applied. Neutron-irradiated tomato seeds were grown in a growth chamber under a $16 \mathrm{~h} \mathrm{light} / 8 \mathrm{~h}$

107 dark illumination time (CHENG \& Edwards 1991). The five-week-old tomato seedlings were

108 infected by TYLCV using a whitefly (Bemisia tabaci) vector. TYLCV infected tomato plants were

109 grown for seven weeks in a growth chamber. The general experimental scheme is drawn in Figure $1101 \mathrm{~A}$.

111

112 Sample collection and total RNA extraction

113 Leaf samples from individual tomato mutants showing TYLCV disease symptoms were harvested 
114 and immediately frozen in liquid nitrogen. Each sample was examined for TYLCV infection by

115 PCR using TYLCV specific primers. Total RNA was extracted using a Qiagen RNeasy Plant Mini

116 Kit (Qiagen GmbH, Hilden, Germany) following the manufacturer's instructions. DNase I was

117 used to digest genomic DNA in the extracted RNA. The quantity and quality of RNA were

118 measured by an Eppendorf BioPhotometer (MedWOW Ltd., Istanbul, Turkey) and agarose gel 119 electrophoresis.

120

121 Library preparation and RNA sequencing

122 The poly(A) RNA libraries were prepared using an NEB Next Ultra RNA Library Prep Kit for 123 Illumina (New England BioLabs Inc., Ipswich, MA, USA) according to manufacturer's 124 instructions. The prepared libraries were analyzed by a 2100 Bioanalyzer instrument (Agilent 125 Genomics, Waldbronn, Germany) to measure quality. A total of eight libraries were paired-end $126(101 \mathrm{bp} \times 2)$ sequenced by an Illumina HiSeq 2000 system at Macrogen, Seoul, Korea. The raw 127 sequenced data from this study were deposited in the SRA database at the National Center for 128 Biotechnology Information with the following accession numbers, SRR6019475-SRR6019483. 129 The detailed information of samples is provided in Table 1.

\section{Assembly of tomato transcripts using RNA-Seq data}

132 All raw data from eight libraries were aligned using Tophat 2.1.1 to the tomato reference genome 133 (ITAG2.4_genomic.fasta) from the International Tomato Genome Sequencing Project (Trapnell et 134 al. 2012). In addition, the Gene Transfer Format (ITAG2.4_gene_models.gff3) file was used for 135 alignment using Tophat resulting in a single BAM file. The BAM file was subjected to Cufflinks 1361.3 .0 (Trapnell et al. 2010) to assemble transcripts. Finally, Cuffcompare was used to annotate 
137 lncRNAs (Trapnell et al. 2010). We obtained assembled transcripts using Cufflinks. The 138 assembled transcripts were subjected to Cuffcompare to annotate the assembled transcripts by 139 comparing the assembled tomato transcripts to the tomato reference annotation. After that, all 140 transcripts including lncRNAs were annotated. Based on annotation, identified lncRNAs were 141 categorized as intergenic regions, antisense, overlapping, and intronic regions.

\section{Identification of tomato IncRNAs}

144

146

147

148

149

150

151

152

153

154

155

156

157

158

159

As shown in Figure 1B, several steps were used to predict novel lncRNAs from the assembled transcripts. First, we extracted assembled transcripts in a fasta format using the gffread program (Weirick et al. 2015). The assembled transcripts were subjected to the Coding Potential Calculator 2 (CPC2) to predict the coding capacity of each transcript with default parameters (Kang et al. 2017). Only transcripts labelled with "noncoding" predicted by CPC2 were included for further analyses. Second, the assembled transcripts were subjected to BLASTX (version 2.2.31) against a tomato protein database with an evalue of 1 e-3 as a cutoff to exclude transcripts with a shared sequence similarity to known tomato proteins. In addition, the transcripts with lengths of less than 200 nt were discarded (Nagata et al. 2004). Third, the transcripts were subjected to PfamScan (version 31.0) with default parameters against the hmmer protein database (version 3.0b2) to identify those containing conserved protein domains ( $\mathrm{Li}$ et al. 2016). Fourth, results from CPC2, PfamScan, and BLASTX were compared to exclude transcripts with coding capacities. Fifth, we excluded transcripts that showed sequence similarity to known miRNA precursors. For this, BLASTN with an evalue of 1e-3 as a cutoff was conducted against the plant miRNA precursors, house-keeping ncRNAs, small nucleolar RNAs (snoRNAs), and non-redundant (NR) protein databases (Liu et al. 2005; Pruitt et al. 2005; Xiao et al. 2009). Finally, we calculated the fragments 
160 per kilobase of transcript per million mapped reads (FPKM) to identify lncRNAs using the Binary 161 version of Sequence Alignment (BWA) followed by the BBMap program with default parameters 162 (Li \& Durbin 2010; Rodríguez-García et al. 2017). The transcripts in which FPKM values were 163 greater than one were considered putative lncRNAs.

Functional classification of IncRNAs and gene ontology (GO) enrichment analysis

To obtain a broad functional overview of identified lncRNAs, we predicted targets of identified lncRNAs by BLASTN search against tomato mRNA sequences using an e-value of $1 \mathrm{e}-5$ as a cutoff. Finally, we selected 794 lncRNAs associated with tomato mRNAs. To predict The selected tomato gene sequences were blasted against Arabidopsis genes (https://www.arabidopsis.org/) to 170 obtain known functions. A majority of tomato genes were converted to the corresponding Arabidopsis locus. Using Arabidopsis loci, we conducted GO enrichment analysis using the GOEAST program with default parameters (Zheng \& Wang 2008). The GO terms obtained for tomato genes possessing lncRNAs were classified according to biological process, molecular function, and cellular component. The REVIGO program was used for visualization of enriched 175 GO terms (Supek et al. 2011).

\section{Expression profiles for 1,563 IncRNAs in response to TYLCV infection}

To establish the expression profiles for the 1,563 lncRNAs in this study, we used previously published RNA-Seq data (PRJNA291401) (Wang et al. 2015). The published RNA-Seq data consisted of three mock and three TYLCV infected samples. The identified 1,563 lncRNAs were used as reference sequences. Raw sequence reads from the published RNA-Seq data were mapped on the 1,563 lncRNAs using Tophat (Trapnell et al. 2012). Transcript assembly was conducted by 
183 Cufflinks. TYLCV infected samples were compared to mock samples to identify differentially 184 expressed genes (DEGs) using Cuffdiff based on a p-value less than 0.05 and a $\log _{2}$ (fold change) 185 greater than one.

186

187

188

189

190

191

192

193

194

195

196

197

198

199

200

201

202

203

204

205

\section{RESULTS}

\section{Assembly of tomato transcripts using eight RNA-Seq data}

To maximize the identification of lncRNAs, we combined all raw data from eight libraries. Raw sequence reads from eight different libraries were subjected to mapping on the reference tomato genome. Of the 128 million reads from eight libraries, almost 117 million (92.2\%) were mapped to the tomato genome, whereas about 11 million (8.8\%) were not mapped (Table 2). The number of mapped reads ranged from $14,824,307$ (30S1) to $16,273,849$ (90D2), whereas the portion of unmapped reads ranged from $7.4 \%(90 \mathrm{D} 2)$ to $12.0 \%(30 \mathrm{~S} 1)$. These mapped reads were used for transcript assembly by Cufflinks, resulting in a total of 39,067 transcripts (Figure 1B).

\section{Identification of tomato IncRNAs}

To identify putative tomato lncRNAs, nine different processes were applied (Figure 1B). First, $19,780(50.63 \%)$ transcripts with coding capacity were excluded by the CPC2 program, and then a BLASTX search against tomato proteins excluded 17,029 (43.58\%) transcripts. In total, 165 transcripts with lengths less than 200 nt were excluded. PfamScan was conducted to exclude three transcripts encoding conserved protein domains. This was followed by a BLASTN search against a miRNA precursor database, which excluded 13 transcripts. In addition, 89 and 10 transcripts were excluded by a BLASTN search for showing sequence similarity to known ncRNAs and snoRNAs, respectively. Finally, the remaining transcripts were subjected to a BLASTX search 
206 against the NR protein database in NCBI, which excluded 553 transcripts. As a result, we obtained

207 1,563 lncRNAs from eight tomato samples (Table S1).

208

209 Classification of identified tomato IncRNAs

210 The lengths of the identified lncRNAs ranged from $201 \mathrm{nt}$ to $4,647 \mathrm{nt}$. About 26\% (416 lncRNAs)

211 of identified lncRNAs were more than 1,000 nt, while 74\% of identified lncRNAs were less than

$2121,000 \mathrm{nt}$. However, most protein coding transcripts (94.3\%) were less than 1,000 nt in size. Among

213 the lncRNAs less than 1,000 nt, lengths ranged from 301 to $800 \mathrm{nt}$ (Figure 2A). We compared the

214 length distribution of identified lncRNAs between our study and a previous study (Wang et al.

215 2015). Both studies showed similar length distribution of identified lncRNAs. In particular, the

216 length of lncRNAs ranged from $400 \mathrm{nt}$ to $500 \mathrm{nt}$ was the highest proportion in both studies. In

217 addition, we examined the number of exons in lncRNAs as well as protein coding transcripts

218 (Figure 2B). Most lncRNAs (90.2\%) were derived from a single exon, and there were two

219 IncRNAs, TCONS_00021506 and TCONS_00021507, derived from six and seven exons,

220 respectively. However, the functions of corresponding genes were unknown. In comparison, half

221 of the protein coding transcripts contain a single exon, whereas $11.7 \%$ of the protein coding

222 transcripts have more than 10 exons.

223 Next, we categorized the identified lncRNAs. Most lncRNAs (49\%) were derived from

224 intergenic regions, followed by antisense lncRNAs (35\%), overlapping (4\%), and intronic regions

$225(3 \%)$ (Figure 2C). Apart from the 769 lncRNAs derived from intergenic regions, 794 lncRNAs

226 were associated with a gene. We further classified the 794 lncRNAs according to chromosome.

227 Five lncRNAs were not assigned to any chromosome. It seems that they were located on random

228 scaffold. With the exception of 110 lncRNAs on chromosome 1, the number of lncRNAs on each 
229 chromosome ranged from 50 (Chromosome 12) to 77 (Chromosome 3), as shown in Figure 2D.

230 The positions of identified lncRNAs on each tomato chromosome are indicated in a circos diagram

231 (Figure 2E). In order to visualize the distribution of identified lncRNAs on different chromosomes,

232 a combination of different graphs including bar plots and volcano plots were used. Interestingly,

233 most lncRNAs were highly enriched at the beginning and the ending of each chromosome. There

234 were relatively few lncRNAs located in the middle of individual chromosome.

235

\section{Expression profiles of identified IncRNAs in eight samples}

237 We examined the expression of 1,563 lncRNAs in eight different samples by calculating the FPKM values. Some lncRNAs were not expressed in eight conditions; however, most lncRNAs were highly expressed. In order to examine the distribution of the expression values for all identified lncRNAs, we generated a box plot using $\log _{10}$ converted FPKM values (Figure 3A). Although standard deviation showed a high degree of difference in each sample, minimum, median, and maximum values among samples did not show significant difference. Next, we conducted PCA analysis to cluster eight conditions based on gene expression of lncRNAs. Our result showed that seven conditions except the condition for soaked sample for $30 \mathrm{~min}$ (30S1) were clustered together (Figure 3B).

We next examined the expression profile of 1,563 lncRNAs in response to TYLCV

247 infection using previously published data (Wang et al. 2015). As a result, 915 lncRNAs out of 1,563 were expressed in mock and TYLCV infected sample (Table S2). A volcano plot showed that the number of up-regulated lncRNAs (19 lncRNAs) was higher than that of down-regulated lncRNAs (11 lncRNAs) (Figure 3C). Expression of 30 differentially expressed lncRNAs were visualized by a heat map (Figure 3D). Unfortunately, the functions of those 30 differentially 
252 expressed lncRNAs are not currently known due to lack of functional studies associated with 253 tomato lncRNAs.

254

255 Functional classification of tomato genes associated with identified IncRNAs

256 Of the 1,563 lncRNAs identified, 794 were associated with tomato genes (Table S3). In order to 257 reveal the functional roles of tomato genes associated with lncRNAs, we examined GO using 258 corresponding Arabidopsis genes. GO enrichment analyses revealed that $47 \mathrm{GO}$ terms (biological 259 process), eight GO terms (cellular component), and 14 GO terms (molecular function) were highly 260 enriched in tomato gene associated lncRNAs (Table S4). In relation to biological processes, GO

261

262

263

264

265

266

267

268

269

270

271

272

273

274

terms associated with positive regulation of the abscisic acid-activated signaling pathway, organic substance metabolism, gluconeogenesis, organelle organization, and cellular catabolism were highly enriched (Figure 3E). Based on cellular component analysis, GO terms related to cytosol, membrane, and cell junction were highly enriched (Figure 3F). Interestingly, according to molecular function, many GO terms were associated with binding. For example, heterocyclic compound binding, phospholipase D activity, and ubiquitin protein ligase binding were frequently identified (Figure 3G).

\section{Identification of target tomato mRNAs of the 1,563 IncRNAs}

GO enrichment analysis revealed that many genes associated with lncRNAs have binding functions important for interaction of lncRNAs with other molecules such as RNAs and proteins. We identified target tomato mRNAs that showed strong sequence similarity to the lncRNAs identified by BLASTN search (Table S5).

Of the lncRNAs identified, 824 showed sequence similarity to at least one tomato mRNA. 
275 For instance, 566 lncRNAs has a single target, whereas two lncRNAs showed sequence similarity

276 to 10 different nucleotide sequences (Figure 4A). The lncRNA TCONS_00000794 (Figure 4B),

277 which was 2,297 nt in length, displayed 10 different regions of six genes. The lncRNA

278 TCONS_00003273, which was 2,081 nt in length, displayed 10 different regions of six genes 279 (Figure 4C).

280 Of 30 differentially expressed lncRNAs, six lncRNAs showed sequence similarity to 281 tomato mRNAs; however, functions of only three corresponding mRNAs are known. For instance, 282 the lncRNA TCONS_00005642, which was down-regulated by TYLCV infection, is associated 283 with BHLH transcription factor (Solyc02g063440.2). Two lncRNAs, TCONS_00020980 and 284 TCONS_00035472, showed sequence similarity to a gene coding pyruvate decarboxylase 2 285 (Solyc06g082140.2) and a gene coding ariadne-like ubiquitin ligase (Solyc11g008590.1), 286 respectively. Both lncRNAs were up-regulated by TYLCV infection.

\section{DISCUSSION}

In this study, we identified tomato lncRNAs that might be associated with at least two different

290 factors. One is neutron irradiation, which causes mutagenesis in the tomato genome, and the other is TYLCV infection, which could change the tomato transcriptome. The irradiated tomato plants

292 showed no significant disease symptoms after TYLCV infection. This result indicates that the genomes of tomato plants could be mutated by neutron irradiation. Thus, the transcriptional

294 regulation in those tomato plants could be changed. We examined lncRNAs to check the change 295 of transcriptome caused by neutron irradiation. Moreover, there were several previous studies identifying lncRNAs in response to TYLCV infection, which facilitates comparison of the 297 lncRNAs among different studies. Since the release of the draft tomato genome sequence 
298 (Consortium 2012), several studies have identified diverse tomato lncRNAs. To date, the identified 299 tomato lncRNAs are related with tomato fruit development (Scarano et al. 2017; Sun \& Xiao 2015; 300 Wang et al. 2018b; Zhu et al. 2015) and TYLCV infection (Wang et al. 2018a; Wang et al. 2015).

301 It seems that fruit development and TYLCV infection, which was included in our study, are two 302 of the most important biological processes and pathogen responses associated with lncRNAs in 303 tomato plants.

304 Compared to two other studies associated with lncRNAs in response to TYLCV infection 305 (Wang et al. 2018a; Wang et al. 2015), the number of lncRNAs identified in our study $(1,563$ 306 lncRNAs) is comparable (1,565 and 2,056 lncRNAs, respectively). A recent study demonstrated 307 that most lncRNAs with low expression are tissue-specific, whereas constitutively expressed 308 lncRNAs are highly conserved in plant species (Deng et al. 2018). Thus, it is important to identify 309 lncRNAs from diverse plant samples because some could be highly regulated by specific 310 environmental factors such as stress conditions, tissues, and developmental stage. For instance, a 311 recent study combining 134 RNA-Seq data has identified 70,635 lncRNAs (Wang et al. 2018b), 312 while the number of lncRNAs identified in other studies ranged from 1,565 (Wang et al. 2015) to 313 10,774 (Scarano et al. 2017).

Although we used a tomato cultivar susceptible to TYLCV, most neutron-irradiated plants showed reduced disease symptoms or no symptoms. Thus, the expression of lncRNAs in our study might be associated with a previous study using a tomato cultivar (CLN2777A) resistant to

317 TYLCV (Wang et al. 2015). The expression profile of the 1,563 lncRNAs in our study using the 318 previous study showed that about 59\% of lncRNAs (915 lncRNAs) were transcribed. We 319 hypothesize cautiously that different genetic backgrounds caused by neutron irradiation change 320 the transcription of several lncRNAs, although both studies performed RNA-Seq followed by 
321

322

323

324

325

326

327

328

329

330

331

332

333

334

335

336

337

338

339

340

341

342

TYLCV infection.

Although RNA-Seq facilitates the identification of numerous lncRNAs in many plant species, only a few lncRNAs were annotated and characterized (Liu et al. 2015). For instance, several studies showed that some lncRNAs are involved in biotic and abiotic stresses (Nejat \& Mantri 2017). An Arabidopsis lncRNA known as ELENA1 interacts with MED19a to regulate PR1 expression functions in increased resistance against Pseudomonas syringae pv tomato DC3000 (Seo et al. 2017). As shown in previous studies, it is very important for lncRNAs to interact with DNAs to form a stable RNA-DNA complex to control the transcriptional activities of target genes (Liu et al. 2015). Previous studies also demonstrated that functions of lncRNAs rely on their binding properties with other nucleic acids and proteins (Marchese et al. 2017; Sun et al. 2017a). For example, many lncRNAs contain several functional regions which are required for interaction with other factors such as ribonucleoproteins and diverse RNA-binding proteins. Similarly, we found that many lncRNA associated mRNAs have binding functions such as nucleotide, small molecule, anion, and histone binding. This result directly suggests that the interaction of lncRNAs with target molecules is an important step in their transcriptional regulation of diverse biological processes, as shown recently (Shi et al. 2017).

Cytoplasm is the place where many lncRNAs are activated (Rashid et al. 2016). Similarly, GO enrichment analysis showed that the lncRNAs in our study were associated with the cytosol and plasma membrane, suggesting that these two cellular components are important places for lncRNAs. In addition, we found that many mRNA targets of lncRNAs were targeted to organelles such as plastids and mitochondria suggesting strong involvements of lncRNAs in organelle biogenesis. A recent study suggests that involvement of lncRNAs not only in nucleus but also in outside of the nucleus (Krause 2018). Of identified biological processes related to target mRNAs 
344 of lncRNAs, functions associated with hormone metabolism, lignin metabolism, developmental

345 processes such as post-embryonic development and developmental process involved in

346 reproduction were highly enriched. Similarly, recent studies also demonstrated the involvement of

347 lncRNAs in metabolisms (An et al. 2018; Lu et al. 2016).

348 Most known lncRNAs were derived from intergenic regions. For example, a previous study

349 showed that $89 \%$ of tomato lncRNAs were derived from intergenic regions, while antisense

350 lncRNAs accounted for only 10\% (Wang et al. 2018b). In contrast, our study revealed that $49 \%$ of

351 lncRNAs was derived from intergenic regions, and antisense lncRNAs accounted for $35 \%$.

352 Moreover, the sizes of the lncRNAs in our study were relatively smaller than that of a previous

353 study (Wang et al. 2015). We hypothesize cautiously that mutagenesis caused by neutron

354 irradiation interferes with the transcriptional regulation of lncRNA expression, resulting in fewer 355 small-sized lncRNAs.

356 Based on sequence similarity BLASTN search, we found several mRNA targets 357 corresponding to identified lncRNAs. Most lncRNAs has a single mRNA target but some lncRNAs

358 have multiple mRNA targets which might be members in the same gene family with strong 359 sequence similarity. Therefore, it is likely that the lncRNAs with multiple target regulate 360 expression of target mRNAs simultaneously.

\section{CONCLUSION}

363 The present study provides a comprehensive bioinformatics analysis of lncRNAs in tomato plants

364 irradiated by neutrons, followed by TYLCV infection. Mutagenesis caused by neutrons influences

365 the transcription of many lncRNAs with shorter lengths and increases the number of antisense

366 lncRNAs. Furthermore, we identified key lncRNAs that are important for TYLCV infection. Based 
367 on these results, the lncRNAs identified in this study provide important resources for

368 characterization of tomato lncRNAs in response to diverse stimuli.

369

370 ADDITIONAL INFORMATION AND DECLARATIONS

371 Funding

372 This work was supported by a National Research Foundation of Korea (NRF) grant funded by the 373 Korean government (MSIT) (No. NRF-2017R1A2B2005117).

374

375 Competing Interests

376 The authors declare that they have no competing interests.

377

378

Author contributions

379 Y.Z., W.K.C., S.L., and S.W.H. designed the research; Y.Z., H.S.B., E.J.K., and V.C. performed 380 the research; Y.Z. and W.K.C. analyzed the data; and Y.Z., W.K.C., S.L., and S.W.H. wrote the 381 paper.

382

383

Data Availability

384 Raw sequence reads derived from eight samples as fastq files were deposited in the NCBI SRA 385 database with respective accession numbers. Most detailed information associated with RNA-Seq 386 data can be accessed in the SRA database with the project number PRJNA403767. 
388

389

390

391

392

394

395

396

397

398

399

400

401

402

403

404

405

406

407

408

409

410

\section{References}

Ahloowalia B, and Maluszynski M. 2001. Induced mutations-A new paradigm in plant breeding. Euphytica 118:167-173.

An N, Fan S, Wang Y, Zhang L, Gao C, Zhang D, and Han M. 2018. Genome-wide identification, characterization and expression analysis of long non-coding RNAs in different tissues of apple. Gene 666:44-57.

Apel K, and Hirt H. 2004. Reactive oxygen species: metabolism, oxidative stress, and signal transduction. Annu Rev Plant Biol 55:373-399.

Bowlt C. 1994. Environmental radioactivity: Measurements and their meaning. Contemporary Physics 35:385-398.

CHENG SH, and Edwards G. 1991. Influence of long photoperiods on plant development and expression of Crassulacean acid metabolism in Mesembryanthemum crystallinum. Plant, Cell \& Environment 14:271-278.

Consortium TG. 2012. The tomato genome sequence provides insights into fleshy fruit evolution. Nature 485:635.

Deng P, Liu S, Nie X, Weining S, and Wu L. 2018. Conservation analysis of long non-coding RNAs in plants. Science China Life Sciences 61:190-198.

Fatica A, and Bozzoni I. 2014. Long non-coding RNAs: new players in cell differentiation and development. Nature Reviews Genetics 15:7-21.

Guo J, and Liu Y-G. 2017. Long non-coding RNAs play an important role in regulating photoperiod-and temperature-sensitive male sterility in rice. Science China Life sciences 60:443. 
411 Ji Y, Schuster DJ, and Scott JW. 2007. Ty-3, a begomovirus resistance locus near the Tomato 412 yellow leaf curl virus resistance locus Ty-1 on chromosome 6 of tomato. Molecular Breeding 20:271-284.

414 Ji Y, Scott JW, Schuster DJ, and Maxwell DP. 2009. Molecular mapping of Ty-4, a new Tomato 415 yellow leaf curl virus resistance locus on chromosome 3 of tomato. Journal of the American Society for Horticultural Science 134:281-288.

417 Kang Y-J, Yang D-C, Kong L, Hou M, Meng Y-Q, Wei L, and Gao G. 2017. CPC2: a fast and accurate coding potential calculator based on sequence intrinsic features. Nucleic acids research 45:W12-W16.

Krause HM. 2018. New and prospective roles for lncRNAs in organelle formation and function. Trends in Genetics.

Lal D. 1987. Cosmogenic nuclides produced in situ in terrestrial solids. Nuclear Instruments and Methods in Physics Research Section B: Beam Interactions with Materials and Atoms 29:238-245.

Li H, and Durbin R. 2010. Fast and accurate long-read alignment with Burrows-Wheeler transform. Bioinformatics 26:589-595. microorganism Chlamydomonas reinhardtii. Scientific reports 6:34109.

Li H, Wang Y, Chen M, Xiao P, Hu C, Zeng Z, Wang C, Wang J, and Hu Z. 2016. Genome-wide long non-coding RNA screening, identification and characterization in a model

Li W, Li C, Li S, and Peng M. 2017. Long noncoding RNAs that respond to Fusarium oxysporum infection in 'Cavendish'banana (Musa acuminata). Scientific reports 7:16939. 
432 Liu C, Bai B, Skogerbø G, Cai L, Deng W, Zhang Y, Bu D, Zhao Y, and Chen R. 2005.

433

434

435

436

437

438

439

440

441

442

443

444

445

446

447

448

449

450

451

452

453 NONCODE: an integrated knowledge database of non-coding RNAs. Nucleic acids research 33:D112-D115.

Liu J, Wang H, and Chua NH. 2015. Long noncoding RNA transcriptome of plants. Plant biotechnology journal 13:319-328.

Lu X, Chen X, Mu M, Wang J, Wang X, Wang D, Yin Z, Fan W, Wang S, and Guo L. 2016. Genome-wide analysis of long noncoding RNAs and their responses to drought stress in cotton (Gossypium hirsutum L.). PLoS One 11:e0156723.

Marchese FP, Raimondi I, and Huarte M. 2017. The multidimensional mechanisms of long noncoding RNA function. Genome biology 18:206.

Mercer TR, Dinger ME, and Mattick JS. 2009. Long non-coding RNAs: insights into functions. Nature Reviews Genetics 10:155-159.

Moriones E, and Navas-Castillo J. 2000. Tomato yellow leaf curl virus, an emerging virus complex causing epidemics worldwide. Virus research 71:123-134.

Nagata T, Iizumi S, Satoh K, Ooka H, Kawai J, Carninci P, Hayashizaki Y, Otomo Y, Murakami K, and Matsubara K. 2004. Comparative analysis of plant and animal calcium signal transduction element using plant full-length cDNA data. Molecular Biology and Evolution 21:1855-1870.

Nejat N, and Mantri N. 2017. Emerging roles of long non-coding RNAs in plant response to biotic and abiotic stresses. Critical Reviews in Biotechnology:1-13.

Polston JE, and Lapidot M. 2007. Management of Tomato yellow leaf curl virus: US and Israel perspectives. Tomato yellow leaf curl virus disease: Springer, 251-262. 
454 Pruitt KD, Tatusova T, and Maglott DR. 2005. NCBI Reference Sequence (RefSeq): a curated 455 non-redundant sequence database of genomes, transcripts and proteins. Nucleic acids 456 research 33:D501-D504.

457

458

459

460

461

462

463

464

465

466

467

468

469

470

471

472

473

474

Qin T, Zhao H, Cui P, Albesher N, and Xiong L. 2017. A nucleus-localized long non-coding RNA enhances drought and salt stress tolerance. Plant physiology:pp. 00574.02017.

Ransohoff JD, Wei Y, and Khavari PA. 2017. The functions and unique features of long intergenic non-coding RNA. Nature Reviews Molecular Cell Biology.

Rashid F, Shah A, and Shan G. 2016. Long non-coding RNAs in the cytoplasm. Genomics, proteomics \& bioinformatics 14:73-80.

Rodríguez-García A, Sola-Landa A, and Barreiro C. 2017. RNA-Seq-Based Comparative Transcriptomics: RNA Preparation and Bioinformatics. Microbial Steroids: Springer, 5972.

Scarano D, Rao R, and Corrado G. 2017. In Silico identification and annotation of non-coding RNAs by RNA-seq and De Novo assembly of the transcriptome of Tomato Fruits. PLoS One 12:e171504.

Seo JS, Sun H-X, Park BS, Huang C-H, Yeh S-D, Jung C, and Chua N-H. 2017. ELF18-INDUCED LONG NONCODING RNA associates with Mediator to enhance expression of innate immune response genes in Arabidopsis. The Plant Cell:tpc. 00886.02016.

Shi W, Quan M, Du Q, and Zhang D. 2017. The interactions between the long non-coding RNA NERDL and its target gene affect wood formation in Populus tomentosa. Frontiers in plant science 8:1035. 
475 Sun X, Ali MSSH, and Moran M. 2017a. The role of interactions of long non-coding RNAs and

476

477

478

479

480

481

482

483

484

485

486

487

488

489

490

491

492

493

494

495

496 heterogeneous nuclear ribonucleoproteins in regulating cellular functions. Biochemical Journal 474:2925-2935.

Sun Y, and Xiao H. 2015. Identification of alternative splicing events by RNA sequencing in early growth tomato fruits. BMC genomics 16:948.

Sun Z, Nair A, Chen X, Prodduturi N, Wang J, and Kocher J-P. 2017b. UClncR: Ultrafast and comprehensive long non-coding RNA detection from RNA-seq. Scientific reports 7:14196.

Supek F, Bošnjak M, Škunca N, and Šmuc T. 2011. REVIGO summarizes and visualizes long lists of gene ontology terms. PLoS One 6:e21800.

Trapnell C, Roberts A, Goff L, Pertea G, Kim D, Kelley DR, Pimentel H, Salzberg SL, Rinn JL, and Pachter L. 2012. Differential gene and transcript expression analysis of RNA-seq experiments with TopHat and Cufflinks. Nature protocols 7:562.

Trapnell C, Williams BA, Pertea G, Mortazavi A, Kwan G, Van Baren MJ, Salzberg SL, Wold BJ, and Pachter L. 2010. Transcript assembly and quantification by RNA-Seq reveals unannotated transcripts and isoform switching during cell differentiation. Nature biotechnology 28:511.

Wang J, Yang Y, Jin L, Ling X, Liu T, Chen T, Ji Y, Yu W, and Zhang B. 2018a. Re-analysis of long non-coding RNAs and prediction of circRNAs reveal their novel roles in susceptible tomato following TYLCV infection. BMC plant biology 18:104.

Wang J, Yu W, Yang Y, Li X, Chen T, Liu T, Ma N, Yang X, Liu R, and Zhang B. 2015. Genomewide analysis of tomato long non-coding RNAs and identification as endogenous target mimic for microRNA in response to TYLCV infection. Scientific reports 5:16946. 
497 Wang M, Zhao W, Gao L, and Zhao L. 2018b. Genome-wide profiling of long non-coding RNAs

498

499

500

501

502

503

504

505

506

507

508

509

510

511

512

513

514

515

516

517 from tomato and a comparison with mRNAs associated with the regulation of fruit ripening. BMC plant biology 18:75.

Weirick T, Militello G, Müller R, John D, Dimmeler S, and Uchida S. 2015. The identification and characterization of novel transcripts from RNA-seq data. Briefings in bioinformatics $17: 678-685$.

Xiao B, Zhang X, Li Y, Tang Z, Yang S, Mu Y, Cui W, Ao H, and Li K. 2009. Identification, bioinformatic analysis and expression profiling of candidate mRNA-like non-coding RNAs in Sus scrofa. Journal of Genetics and Genomics 36:695-702.

Zamir D, Ekstein-Michelson I, Zakay Y, Navot N, Zeidan M, Sarfatti M, Eshed Y, Harel E, Pleban T, and Van-Oss H. 1994. Mapping and introgression of a tomato yellow leaf curl virus tolerance gene, Ty-1. Theoretical and Applied Genetics 88:141-146.

Zheng Q, and Wang X-J. 2008. GOEAST: a web-based software toolkit for Gene Ontology enrichment analysis. Nucleic acids research 36:W358-W363.

Zheng Y, Wang Y, Ding B, and Fei Z. 2017. Comprehensive Transcriptome Analyses Reveal that Potato Spindle Tuber Viroid Triggers Genome-Wide Changes in Alternative Splicing, Inducible trans-Acting Activity of Phased Secondary Small Interfering RNAs, and Immune Responses. Journal of Virology 91:e00247-00217.

Zhu B, Yang Y, Li R, Fu D, Wen L, Luo Y, and Zhu H. 2015. RNA sequencing and functional analysis implicate the regulatory role of long non-coding RNAs in tomato fruit ripening. Journal of experimental botany 66:4483-4495. 
518 Zhu M, Zhang M, Xing L, Li W, Jiang H, Wang L, and Xu M. 2017. Transcriptomic analysis of

519 long non-coding RNAs and coding genes uncovers a complex regulatory network that is $520 \quad$ involved in maize seed development. Genes 8:274.

521 Zhu QH, Stephen S, Taylor J, Helliwell CA, and Wang MB. 2014. Long noncoding RNA s 522 responsive to $\mathrm{F}$ usarium oxysporum infection in A rabidopsis thaliana. New phytologist $523 \quad 201: 574-584$.

524

525 


\section{Figure legends}

527 Figure 1. Schematic workflow to identify tomato lncRNAs.

528 (A) Experimental workflow for neutron irradiation, TYLCV infection, and RNA-Seq. The detailed

529 experimental processes have been shown. First, tomato seeds were divided into two groups. The 530 dry and soaked seeds were placed in distilled water for eight hours before being submitted to 531 neutron irradiation for 30 and 90 minutes, respectively. Second, after irradiation, the seeds were 532 cultivated in a growth chamber and then infected by TYLCV using whitefly. Third, total RNAs 533 were extracted from samples, followed by library preparation and RNA sequencing. (B) 534 Bioinformatic procedures to identify lncRNAs using RNA-Seq data. Paired-end sequence data 535 from eight libraries were subjected to transcript assembly using Tophat and Cufflinks. For each 536 step, the number of excluded transcripts was provided. Full nucleotide sequences and detailed 537 information for 1,563 lncRNAs can be found on the figshare website 538 (https://doi.org/10.6084/m9.figshare.5914396.v1).

540 Figure 2. Classification of identified tomato lncRNAs.

541 (A) Size distributions of identified lncRNAs and protein-coding transcripts in this study are

542 visualized by green and red bars, respectively. (B) The proportions of exons associated with 543 lncRNAs and protein-coding transcripts are indicated by blue and orange bars, respectively. (C) 544 Categories of identified lncRNAs. (D) Number of lncRNAs identified on each tomato 545 chromosome. Chromosomal distribution of the identified lncRNAs except those derived from 546 intergenic regions. (E) Four different plot types including the rectangles, scatter, line, and area 547 were used to visualize chromosomal positions of identified lncRNAs by a circos diagram. 
549 Figure 3. Expression profiles of identified lncRNAs and their functional classification.

550 (A) Box plot showing the overall distribution of lncRNAs expression $\left(\log _{10}\right.$ converted FPKM

551 values) in eight samples. (B) PCA analysis of eight conditions based on the expression of

552 lncRNAs. FPKM values of lncRNAs in eight conditions were subjected to PCA analysis using

553 ClustVis program (https://biit.cs.ut.ee/clustvis/). (C) Volcano plot illustrating the distribution of p-

554 values and fold changes for expression of lncRNAs in response to TYLCV infection compared to

555 mock samples. Blue colored dots indicate differentially expressed genes (DEGs). (D) The

556 expression levels of differentially expressed lncRNAs in response to lncRNAs visualized by a heat

557 map. Enriched GO terms of the identified lncRNAs based on biological process (E), cellular

558 component $(\mathrm{F})$, and molecular function $(\mathrm{G})$.

559

560 Figure 4. Identification of target mRNAs for the identified lncRNAs by sequence similarity.

561 (A) Number of target mRNAs for individual lncRNA revealed by a BLASTN search. The possible

562 interactions of mRNAs with two selected lncRNAs, TCONS_00000794 (B) and

563 TCONS_00003273 (C). Some lncRNAs showed sequence similarity to two regions of a mRNA

564 indicated by 1 and 2 .

565

566 Supplementary data

567 Table S1. Detailed information of the 1,563 identified lncRNAs.

568 For each identified lncRNA, detailed information including identity number, chromosome, RNA

569 strand, position on chromosome, length, and FPKM value in each sample are provided.

570

571 Table S2. Expression profile of identified lncRNAs in response to TYLCV infection. 
572 Expression profile of the identified lncRNAs in response to TYLCV infection was obtained. Green

573 and red colors indicate down-regulated and up-regulated lncRNAs, respectively, which were

574 selected by a p-value less than 0.05 and a $\log 2$ (fold change) more than 1.

575

576 Table S3. List of tomato lncRNAs associated with mRNAs and their respective functions.

577

578 Table S4. Highly enriched gene ontology (GO) terms for tomato mRNAs associated with 579 lncRNAs. Identification of GO enrichment information of identified lncRNAs.

580

581 Table S5. List of target mRNAs for identified lncRNAs.

582 Target mRNAs for each identified lncRNA are listed with blast results.

583

584 


\section{Table $\mathbf{1}$ (on next page)}

Sample descriptions for identification of IncRNA from eight different tomato mutants in response to TYLCV infection. 
1 Table 1 Sample descriptions for identification of lncRNA from eight different tomato mutants in response to TYLCV infection.

\begin{tabular}{|c|c|c|c|c|c|c|c|}
\hline Sample & $\begin{array}{l}\text { Seed } \\
\text { condition }\end{array}$ & $\begin{array}{l}\text { Neutron irradiation } \\
\text { time }\end{array}$ & $\begin{array}{l}\text { TYLCV } \\
\text { infection }\end{array}$ & Protocol 1 & Protocol 2 & Protocol 3 & Data \\
\hline 30D1 & Dry & $30 \mathrm{~min}$ & $\begin{array}{l}\text { TYLCV } \\
\text { infected }\end{array}$ & $\begin{array}{l}\text { Leaf } \\
\text { tissues }\end{array}$ & $\begin{array}{l}\text { RNA } \\
\text { extraction }\end{array}$ & $\begin{array}{l}\text { Library preparation and } \\
\text { RNA-Seq }\end{array}$ & SRR6019475 \\
\hline $30 \mathrm{D} 2$ & Dry & $30 \mathrm{~min}$ & $\begin{array}{l}\text { TYLCV } \\
\text { infected }\end{array}$ & $\begin{array}{l}\text { Leaf } \\
\text { tissues }\end{array}$ & $\begin{array}{l}\text { RNA } \\
\text { extraction }\end{array}$ & $\begin{array}{l}\text { Library preparation and } \\
\text { RNA-Seq }\end{array}$ & SRR6019476 \\
\hline $30 \mathrm{~S} 1$ & Soaked & $30 \mathrm{~min}$ & $\begin{array}{l}\text { TYLCV } \\
\text { infected }\end{array}$ & $\begin{array}{l}\text { Leaf } \\
\text { tissues }\end{array}$ & $\begin{array}{l}\text { RNA } \\
\text { extraction }\end{array}$ & $\begin{array}{l}\text { Library preparation and } \\
\text { RNA-Seq }\end{array}$ & SRR6019477 \\
\hline $30 \mathrm{~S} 2$ & Soaked & $30 \mathrm{~min}$ & $\begin{array}{l}\text { TYLCV } \\
\text { infected }\end{array}$ & $\begin{array}{l}\text { Leaf } \\
\text { tissues }\end{array}$ & $\begin{array}{l}\text { RNA } \\
\text { extraction }\end{array}$ & $\begin{array}{l}\text { Library preparation and } \\
\text { RNA-Seq }\end{array}$ & SRR6019478 \\
\hline 90D1 & Dry & $90 \mathrm{~min}$ & $\begin{array}{l}\text { TYLCV } \\
\text { infected }\end{array}$ & $\begin{array}{l}\text { Leaf } \\
\text { tissues }\end{array}$ & $\begin{array}{l}\text { RNA } \\
\text { extraction }\end{array}$ & $\begin{array}{l}\text { Library preparation and } \\
\text { RNA-Seq }\end{array}$ & SRR6019479 \\
\hline 90D2 & Dry & $90 \mathrm{~min}$ & $\begin{array}{l}\text { TYLCV } \\
\text { infected }\end{array}$ & $\begin{array}{l}\text { Leaf } \\
\text { tissues }\end{array}$ & $\begin{array}{l}\text { RNA } \\
\text { extraction }\end{array}$ & $\begin{array}{l}\text { Library preparation and } \\
\text { RNA-Seq }\end{array}$ & SRR6019480 \\
\hline $90 \mathrm{~S} 1$ & Soaked & $90 \mathrm{~min}$ & $\begin{array}{l}\text { TYLCV } \\
\text { infected }\end{array}$ & $\begin{array}{l}\text { Leaf } \\
\text { tissues }\end{array}$ & $\begin{array}{l}\text { RNA } \\
\text { extraction }\end{array}$ & $\begin{array}{l}\text { Library preparation and } \\
\text { RNA-Seq }\end{array}$ & SRR6019481 \\
\hline $90 \mathrm{~S} 2$ & Soaked & $90 \mathrm{~min}$ & $\begin{array}{l}\text { TYLCV } \\
\text { infected }\end{array}$ & $\begin{array}{l}\text { Leaf } \\
\text { tissues }\end{array}$ & $\begin{array}{l}\text { RNA } \\
\text { extraction }\end{array}$ & $\begin{array}{l}\text { Library preparation and } \\
\text { RNA-Seq }\end{array}$ & SRR6019482 \\
\hline
\end{tabular}


Table 2 (on next page)

Summary of sequence alignment for eight libraries from the tomato genome. 
1 Table 2 Summary of sequence alignment for eight libraries from the tomato genome.

\begin{tabular}{cccc}
\hline Library name & No. of raw sequence reads & No. of unmapped reads & No. of mapped reads \\
\hline 30D1 & $15,363,905$ & $1,200,998(7.8 \%)$ & $14,162,907(92.2 \%)$ \\
30D2 & $16,660,349$ & $1,446,788(8.7 \%)$ & $15,213,561(91.3 \%)$ \\
30S1 & $16,851,416$ & $2,027,109(12.0 \%)$ & $14,824,307(88.0 \%)$ \\
30S2 & $16,342,941$ & $1,432.998(8.8 \%)$ & $14,909,943(91.2 \%)$ \\
90D1 & $14,248,247$ & $1,079,660(7.6 \%)$ & $13,168,587(92.4 \%)$ \\
90D2 & $17,578,965$ & $1,305,116(7.4 \%)$ & $16,273,849(92.6 \%)$ \\
90S1 & $15,600,291$ & $1,586,768(10.2 \%)$ & $14,013,523(89.8 \%)$ \\
90S2 & $16,239,706$ & $1,264,170(7.8 \%)$ & $14,975,536(92.2 \%)$ \\
Total & $128,885,820$ & $11,343,607(8.8 \%)$ & $117,542,213(92.2 \%)$ \\
\hline
\end{tabular}

2 


\section{Figure 1 (on next page)}

Schematicworkflow to identify tomato IncRNAs.

(A) Experimental workflow for neutron irradiation, TYLCV infection, and RNA-Seq. The detailed experimental processes have been shown. First, tomato seeds were divided into two groups. The dry and soaked seeds were placed in distilled water for eight hours before being submitted to neutron irradiation for 30 and 90 minutes, respectively. Second, after irradiation, the seeds were cultivated in a green chamber and then infected by TYLCV using whitefly. Third, total RNAs were extracted from samples, followed by library preparation and RNA sequencing. (B) Bioinformatic procedures to identify IncRNAs using RNA-Seq data. Paired-end sequence data from eight libraries were subjected to transcript assembly using Tophat and Cufflinks. For each step, the number of excluded transcripts was provided. Full nucleotide sequences and detailed information for 1,563 IncRNAs can be found on the figshare website (https://doi.org/10.6084/m9.figshare.5914396.v1). 
A

\section{B Manuscript to be reviewed}
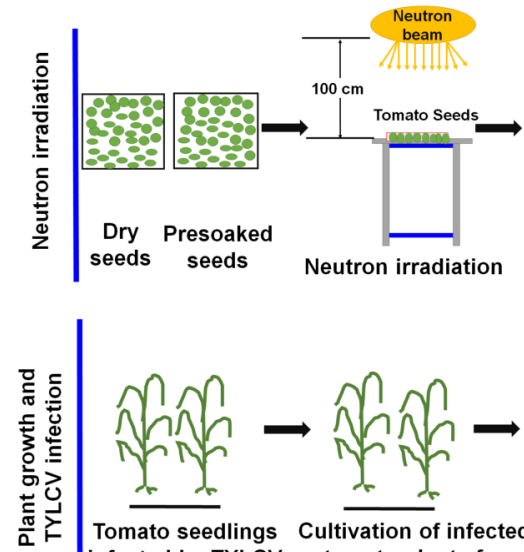
Tomato seedlings
infected by TYLCV via whitefly

Cultivation of infected Collection of tomato plants for seven weeks

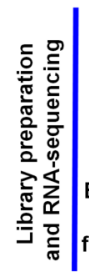

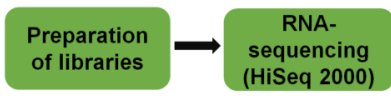

Paired-end

Extraction of total Preparation of eight RNAs

from leaf samples libraries for RNA-sequencing

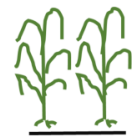

Cultivation in a growth chamber

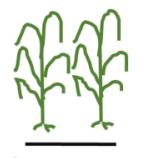
leaf samples sequencing using HiSeq2000 system

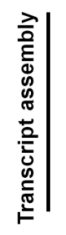
cufflinks cuffcompare CPC2

\section{Alignment of RNA-Seq data}

Assembly of transcripts by

Annotation of transcripts by

Prediction of coding capacity by

\section{BLASTX against tomato proteins}

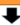

Selection of transcripts $>200 \mathrm{nt}$ in length

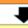

Identification of conserved domain by PfamScan

$$
\checkmark
$$

BLASTN against miRNA precursors

$\checkmark$

BLASTN against known ncRNAs

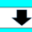

BLASTN against known snoRNAs

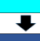

BLATX against NR database (7)

Calculation of FPKM values
39,067 transcripts

Exclude 19,780 transcripts

Exclude 17,029 transcripts

Exclude 165 transcripts

Exclude 3 transcripts

Exclude 13 transcripts

Exclude 89 transcripts

Exclude 10 transcripts

Exclude 553 transcripts

1,563 IncRNA candidates 


\section{Figure 2 (on next page)}

Classificationof identified tomato IncRNAs.

(A) Size distributions of identified IncRNAs and protein-coding transcripts in this study are visualized by green and red bars, respectively. (B) The proportions of exons associated with IncRNAs and protein-coding transcripts are indicated by blue and orange bars, respectively. (C) Categories of identified IncRNAs. (D) Number of IncRNAs identified on each tomato chromosome. Chromosomal distribution of the identified IncRNAs except those derived from intergenic regions. (E) Chromosomal positions of identified IncRNAs visualized by a circos diagram. 


\section{Figure $\mathbf{3}$ (on next page)}

Expression profiles of identified IncRNAs and their functional classification.

(A) Box plot showing the overall distribution of IncRNAs expression (log10 converted FPKM values) in eight samples. (B) PCA analysis of eight conditions based on the expression of IncRNAs. FPKM values of IncRNAs in eight conditions were subjected to PCA analysis using ClustVis program ( https://biit.cs.ut.ee/clustvis/ ). (C) Volcano plot illustrating the distribution of $p$-values and fold changes for expression of IncRNAs in response to TYLCV infection compared to mock samples. Blue colored dots indicate differentially expressed genes (DEGs). (D) The expression levels of differentially expressed IncRNAs in response to IncRNAs visualized by a heat map. Enriched GO terms of the identified IncRNAs based on biological process (E), cellular component (F), and molecular function (G). 


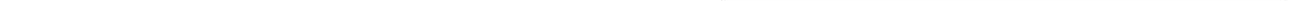




\section{Figure 4 (on next page)}

Identification oftarget mRNAs for the identified IncRNAs by sequence similarity.

(A) Number of target mRNAs for individual IncRNA revealed by a BLASTN search. The possible interactions of mRNAs with two selected IncRNAs, TCONS_00000794 (B) and TCONS_00003273 (C). Some IncRNAs showed sequence similarity to two regions of a mRNA indicated by 1 and 2 . 
